\title{
Effectiveness evaluation of data link based on the improved grey interval correlation degree
}

\author{
Tao $\mathrm{Jia}^{1}$, Weibin Zhao ${ }^{2, \text { a }}$ and Guoqing Huang ${ }^{1,2}$ \\ ${ }^{1}$ The First Aeronautical College of Air Force, Xinyang 464000, China \\ ${ }^{2}$ School of Information Engineering, Zhengzhou University, Zhengzhou 450001, China
}

\begin{abstract}
To solve the problem of the fuzzy multiple attribute decision making, the improved grey interval correlation degree was proposed. In the improved method, the attribute values took the form of the interval numbers; it proposed an algorithm for comparing the size of interval numbers and used the combination weighting method to make the weights more reasonable. To evaluate the effectiveness of the system, the concrete steps of the improved method were given. It used analytic hierarchy process (AHP) and Delphi method to establish the scientific and reasonable index system of data link. An example was analyzed and the results show that the improved method is reasonable and feasible. The improved method can be used to rank the limited projects and select the optimal one through dealing with the interval numbers.
\end{abstract}

Keywords: data link; index system; grey interval correlation degree; effectiveness evaluation; combination weighting.

\section{Introduction}

Grey correlation degree is an important part of grey system theory, which is used to quantitatively analyze the relationship between system sequences [1]. The grey correlation comprehensive optimization method makes the evaluation result more reasonable by considering both the optimal reference scheme and the worst scheme [2-3]. Grey correlation analysis is used to determine the judgment matrix in the ANP method, which overcomes the subjectivity and uncertainty of the weight determined by expert scoring [4].The index is weighted according to the important degree of the index, which improves the credibility of the results [5]. The gray correlation analysis is used to optimize the green production project, which can obtain the lean output with the minimum energy consumption [6] and so on.

In the above researches, the attribute values are precise numbers, but because of the fuzziness of human thought and the complexity of the objective things, it is often difficult for people to give precise numbers in decision-making process [7-10]. Interval number is more able to reflect the reliability of the fuzzy numbers. Therefore, in order to rank all the limited projects and select the optimal one through a certain way with the fuzzy interval numbers, the improved grey interval correlation degree method was proposed for effectiveness evaluation.

a Corresponding author : zzuweibin@163.com

(C) 2016. The authors - Published by Atlantis Press 


\section{The improved method of grey interval correlation degree for effectiveness evaluation}

The improved method establishes the hierarchical relationship based on the AHP and Delphi method. The attribute values are interval numbers, it improves the accuracy of the data source by considering the influence of subjective and objective fuzziness on the evaluation results. Weights are determined by using the analytic hierarchy process and an improved maximizing deviation method, the combination weighting method is used to determine the weight, it is not only to consider the preference of the experts to the index, but also to reduce the subjective arbitrary of the weight. The information vector of interval number is extracted [9]. The vector similarity algorithm is used as the grey correlation degree model to calculate the correlation degree between the information vector of the comparison scheme and the reference scheme. It obtains the relational sequence of each scheme according to the numerical value of the correlation degree.

According to the above improvement ideas, the concrete steps of using the improved grey interval correlation method to evaluate the effectiveness are as follows:

Step 1: establish index system.

Delphi method uses the knowledge and experience of experts to determine the indicators of the complex system. When the index system is established, the complex problem is usually decomposed into some easily measurable indicators. The set of comparison schemes is denoted as $A=\{A 1, A 2$, $A 3, \ldots, A n\}$; The set of indicators in the criteria layer is denoted as $B=\{B 1, B 2, \ldots, B f\}$; The set of indicators in the index layer is denoted as $\mathrm{C}=\{\mathrm{C} 1, \mathrm{C} 2, \ldots, \mathrm{Cm}\}$.

Step 2: get and normalize the interval number matrix.

(1) Quantitative indicators

The interval number of the corresponding index value of the index layer $c_{j}$ in the scheme $A_{i}$ is

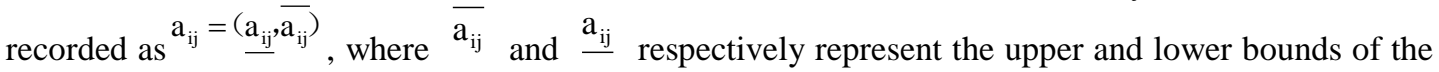
interval.

(2) Qualitative indicators

The remark set of experts is determined as \{excellent, good, general, poor, very poor and the interval number corresponding to the remark set is denoted as $\{[0.9,1.0],[0.7,0.8],[0.5,0.6],[0.3$, $0.4],[0.1,0.2]\}$. We determine the interval number of qualitative indicators according to the maximum degree of membership. The interval number matrix of the index value is denoted as $\mathrm{X}=$ $\left(\mathrm{a}_{\mathrm{ij}}\right)_{\mathrm{n} \times \mathrm{m}}$.

For the quantitative indicators, the physical meanings of each index are not necessarily the same; the mathematical dimension is not always the same. There is a big gap between the data and there is no comparability. It is difficult to get the correct results if the data are not normalized. Therefore, the original index values must be eliminated the dimension and transformed to comparable data. For benefit index and cost index, the formula (1) and formula (2) are used to normalize the data separately to make each index value in the range of $[0,1]$.

$$
\begin{aligned}
b_{i j} & =\frac{a_{i j}}{\max _{i=1}^{n}\left(\overline{a_{i j}}\right)} . \\
b_{i j} & =\frac{\min _{i=1}^{n}\left(a_{i j}\right)}{a_{i j}} .
\end{aligned}
$$

The normalized matrix is $Y=\left(b_{i j}\right)_{n \times m}$, where $b_{i j}=\left(b_{i j} \overline{b_{i j}}\right)$.

Step 3: determine the weight of the index.

(1)The determination of the subjective weight 
The subjective weight is determined by the method of AHP. According to the importance of each index with respect to its upper level, pairwise comparison is made to get the weight judgment matrix $\mathrm{C}=(\mathrm{cij}) \mathrm{m} \times \mathrm{m}$. The maximum eigenvalue of the judgment matrix is obtained, which is used to test the consistency of the weight judgment matrix. When the consistency check probability satisfy with $\mathrm{CR}<0.1$, the judged matrix meet the consistency. By the formula (3), (4), (5) to get the subjective weight wsc $=($ ws1, ws2,...,wsm). Otherwise, a renewed pairwise comparison is needed to determine the weight judgment matrix.

$$
\begin{gathered}
\mathrm{M}_{\mathrm{i}}=\sum_{\mathrm{j}=1}^{\mathrm{m}} \mathrm{c}_{\mathrm{ij}}, \quad \mathrm{i}=1,2, \ldots, \mathrm{m} \\
\overline{\mathrm{w}_{\mathrm{si}}}=\frac{\mathrm{M}_{\mathrm{i}}}{\mathrm{m}}, \quad \mathrm{i}=1,2, \ldots, \mathrm{m} \\
\mathrm{w}_{\mathrm{si}}=\frac{\overline{\mathrm{w}_{\mathrm{si}}}}{\sum_{\mathrm{i}=1}^{\mathrm{m}} \overline{\mathrm{w}_{\mathrm{si}}}}, \quad \mathrm{i}=1,2, \ldots, \mathrm{m}
\end{gathered}
$$

(2)The determination of objective weight

Objective weight is determined by the method of maximizing deviation. The core idea of the method is that the maximum difference between the index values of the different schemes for the same index can play an important influence on the evaluation results, The greater the index value of the scheme, the greater the weight of the index. In order to deal with the interval number, the formula (6) is proposed to calculate the objective weight of the index.

$$
\mathrm{w}_{\mathrm{oc}}=\frac{\sum_{\mathrm{i}=1}^{\mathrm{n}} \sum_{k=1}^{n}\left(\left|\overline{\mathrm{b}_{\mathrm{ic}}}-\underline{\mathrm{b}_{\mathrm{kc}}}\right|+\left|\underline{\mathrm{b}_{\mathrm{ic}}}-\overline{\mathrm{b}_{\mathrm{kc}}}\right|\right)}{\sum_{\mathrm{c}=1}^{\mathrm{j}} \sum_{\mathrm{i}=1}^{\mathrm{n}} \sum_{k=1}^{n}\left(\left|\overline{\mathrm{b}_{\mathrm{ic}}}-\underline{\mathrm{b}_{\mathrm{kc}}}\right|+\left|\underline{\mathrm{b}_{\mathrm{ic}}}-\overline{\mathrm{b}_{\mathrm{kc}}}\right|\right)} .
$$

The objective weight of each index is recorded as $\mathrm{w}_{\mathrm{oc}}=\left(\mathrm{w}_{\mathrm{o} 1}, \mathrm{w}_{\mathrm{o} 2}, \ldots, \mathrm{w}_{\mathrm{om}}\right)$.

(3)Combination weighting

$$
\mathrm{w}_{\mathrm{c}}=\beta \mathrm{w}_{\mathrm{sc}}+(1-\beta) \mathrm{w}_{\mathrm{oc}}
$$

By formula (7), the combined weights are calculated. $w_{c}$ is satisfied $0 \leq w_{c} \leq 1, \sum_{c=1}^{m} w_{c}=1$. We can select the coefficient $\beta$ according to the importance of the subjective and objective weight. In this paper, the coefficient $\beta$ is 0.5 .

Step 4: determine the interval number of the scheme.

For interval number $\mathrm{b}_{\mathrm{ij}}=\left(\mathrm{b}_{\mathrm{ij}}, \overline{\mathrm{b}_{\mathrm{ij}}}\right)$ and its index weight $\mathrm{w}$, we can weight the index interval number by the formula (8) and to determine the interval number $\mathrm{d}$ of the scheme A by the formula (9).

$$
\begin{gathered}
\mathrm{c}_{\mathrm{ij}}=\mathrm{w}_{\mathrm{ij}} \mathrm{b}_{\mathrm{ij}}=\left(\mathrm{w}_{\mathrm{ij}} \underline{\mathrm{b}_{\mathrm{ij}},} \mathrm{w}_{\mathrm{ij}} \overline{\mathrm{b}_{\mathrm{ij}}}\right) . \\
\mathrm{d}=\mathrm{w}_{1}\left(\mathrm{c}_{11}+\mathrm{c}_{12}+\cdots+\mathrm{c}_{1 \mathrm{e}}\right)+\mathrm{w}_{2}\left(\mathrm{c}_{21}+\mathrm{c}_{22}+\cdots+\mathrm{c}_{2 \mathrm{~g}}\right)+\cdots+\mathrm{w}_{\mathrm{f}}\left(\mathrm{c}_{\mathrm{f} 1}+\mathrm{c}_{\mathrm{f} 2}+\cdots+\mathrm{c}_{\mathrm{fh}}\right) .
\end{gathered}
$$

Step 5: extract the information vector of interval number 
For interval numbers $\mathrm{d}=(\underline{\mathrm{d}}, \overline{\mathrm{d}})$, the interval number information vector [9] $I_{\mathrm{d}}=\left(\mathrm{m}_{\mathrm{d}, \mathrm{z}_{\mathrm{d}}}\right)$ is extracted by the formula (10) and (11).

$$
\begin{aligned}
& \mathrm{m}_{\mathrm{d}}=\frac{\mathrm{d}+\overline{\mathrm{d}}}{2} . \\
& \mathrm{z}_{\mathrm{d}}=\frac{\overline{\mathrm{d}}-\underline{\mathrm{d}}}{2} .
\end{aligned}
$$

Step 6: determine the reference scheme, calculate the correlation degree

The ideal situation is generally regarded as the reference scheme, the principle of which is to choose the optimal value from the existing data as the reference vector. The information vector of the reference scheme is denoted as $\mathrm{I}_{\mathrm{cd}}=\left(\mathrm{m}_{\mathrm{cd}}, \mathrm{z}_{\mathrm{cd}}\right)$ and $\mathrm{m}_{\mathrm{cd}}=\max \left(\mathrm{m}_{\mathrm{d}}^{\mathrm{n}}\right), \mathrm{z}_{\mathrm{cd}}=\min \left(\mathrm{z}_{\mathrm{d}}^{\mathrm{n}}\right)$. The correlation degree is calculated by the formula (12) and (13) [12].

$$
\begin{aligned}
& \mathrm{d}\left(I_{\mathrm{d}}, I_{\mathrm{cd}}\right)=\sqrt{\sum_{\mathrm{i}=1}^{2}\left(I_{\mathrm{cd} i}-I_{\mathrm{d} i}\right)^{2}} . \\
& \mathrm{r}\left(I_{\mathrm{d}}, I_{\mathrm{cd}}\right)=\frac{\alpha}{\alpha+\mathrm{d}\left(I_{\mathrm{d}}, I_{\mathrm{cd}}\right)} .
\end{aligned}
$$

In formula (13), $\alpha$ express the resolution coefficient and $\alpha>0$

\section{Index system of data link}

In the modern local war, data link effectively make weapons platform and command platform combine to improve the overall operational capability of the weapon with its unique advantages of battlefield sharing, accurate command control and the seamless connection of weapon coordination, accurately grasp the characteristics of information warfare [13-16].The data link is in the central position in the modern cooperative operations, in the actual combat. The data link is affected by many factors and the logical relation is complex in the process of the situation awareness, cooperative attack. Therefore, it is very difficult to evaluate the effectiveness of the data link. The scientific and reasonable index system is the basis for the evaluation of the data link. Based on the AHP method and the Delphi method, a comprehensive index system of data link was established. The index system was determined as shown in figure 1.

The effectiveness of data link is divided into four criteria layer indicators, which are situational awareness ability, data fusion ability, anti-interference ability and survival ability. Situational awareness ability is a real-time grasp of the number, position, speed, track and other information of the target, through cooperative reconnaissance of reconnaissance aircraft, fighter aircraft and ground command units. Situational awareness ability is divided into five underlying indicators, which are network compatibility, communication coverage, communication capacity, real-time performance, task allocation capacity. Data fusion ability is the ability to carry out the correct and fast transmission and process of situational information, it divided into five underlying indicators, which are data transmission rate, data transmission delay, data reliability, data update rate and data processing accuracy. The anti-interference ability reflects the measures taken by the data link in the complex electromagnetic environment, it is divided into confidentiality and networking mode. Survival ability is the degree that the data link can be repaired quickly and can still keep the original working condition after being attacked in the actual combat environment, it is divided into equipment reliability, maintainability and backup channel. 


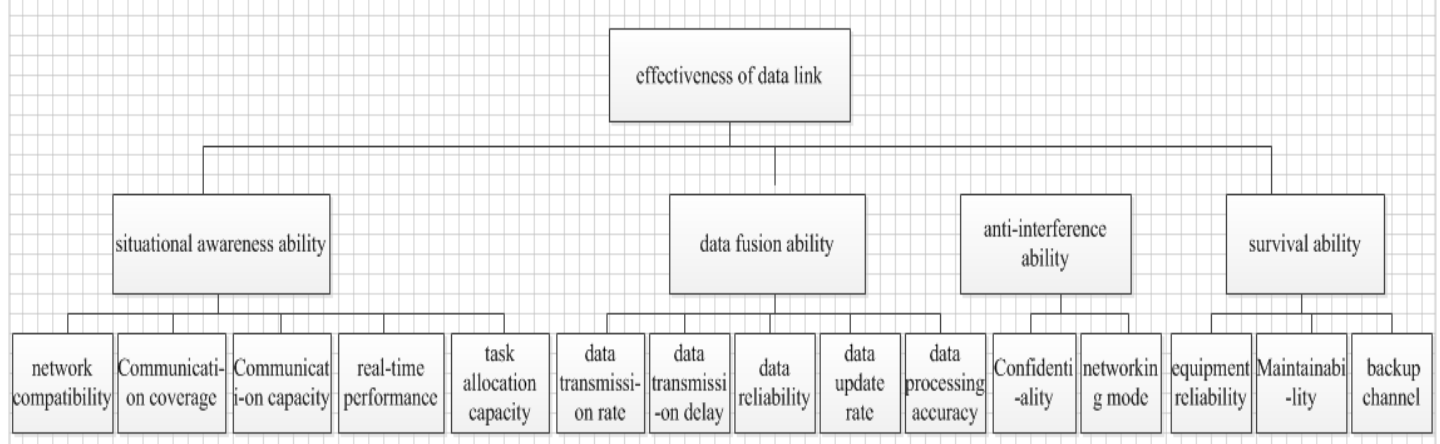

Figure 1 .The index frame of data link

\section{Case analysis}

In this paper, we use schemes and data provided in the literature [17-19] to carry on the case analysis. It is to evaluate the effectiveness of the data link L1 and data link L2.

1) Obtain the original data matrix of the index system. The interval number of the qualitative index is determined according to the principle of the maximum membership degree; for quantitative indicators, the optimal and worst values of each index are determined by calculating or measuring the number of the indicators.

2) The interval numbers in the original data matrix are normalized separately to make each index value in the range of $[0,1]$ in accordance with the formula (1) or (2). The normalized matrix is:

$$
\begin{gathered}
X=\left[\begin{array}{ccccccc}
(0.9,1.0) & (0.5,0.88) & (0.9,1.0) & (0.74,1.0) & (0.7,0.8) & (0.50,0.75)(0.55,1.0)(0.86,1.0) \\
(0.9,1.0) & (0.7,1.0) & (0.8,0.9) & (0.36,0.49) & (0.9,1.0) & (0.70,1.0)(0.36,0.76)(0.92,1.0) \\
(1.0,1.0) & (0.89,1.0) & (0.9,1.0) & (0.9,1.0) & (0.9,1.0) & (0.9,1.0)(0.9,1.0) \\
(0.75,0.75) & (0.89,1.0) & (0.7,0.8) & (0.9,1.0) & (0.7,0.8) & (0.9,1.0)(0.9,1.0)
\end{array}\right]
\end{gathered}
$$

3) Using the combination weighting method to determine the index weight $\mathrm{w} 1=(0.0954,0.2139,0.1572,0.3536,0.1799), \mathrm{w} 2=(0.1063,0.2806,0.1531,0.2046,0.2554)$ $\mathrm{w} 3=(0.6521,0.3479), \mathrm{w} 4=(0.6415,0.2362,0.1223), \mathrm{w}=(0.4032,0.2607,0.1275,0.2086)$.

4) Calculate the interval number of the comparison schemes according to the formula (8), (9). $\mathrm{d}_{\mathrm{L} 1}=(0.79456688520,0.96821542390) ; \mathrm{d}_{\mathrm{L} 2}=(0.70311901300,0.84666690500)$.

$5)$ The interval number information vector is extracted by the formula (10) and (11).

$\mathrm{I}_{\mathrm{dL} 1}=$ (0.881391154550, 0.086824269350); $\mathrm{I}_{\mathrm{dL} 2}=(0.774892959000,0.071773946000)$

6) The information vector of the reference scheme is $I_{c d}=(0.88139115455,0.07177394600)$. The correlation degree are calculated by the formula (12) and (13) $r_{L 1}=0.970778926509337$; $\mathrm{r}_{\mathrm{L} 2}=0.8244047610835$.

7) Compositor according to the value of the relational degree, the data link L1 is better than the data link L2 due to rL1>rL2. It is consistent with reference [19].

\section{Conclusions}

In this paper, the improved grey interval correlation degree method is proposed, and through analyzing the requirement of the data link in the actual combat, the scientific and reasonable index system of the data link was set up. Because of the interval number used by the improved method is more reliable in the fuzzy multiple attribute decision making and the weights determined by the combination weighting method are more reasonable, the evaluation result of the data link is more credible. The improved method can be used for fuzzy multiple attribute decision making. 


\section{References}

1. Zhang Jie, Tang Hong, Su Kai. "Research on the methods of effectiveness evaluation”. Beijing, National Defense Industry Press, (2009)

2. Wang Hao, Ji Yongqing, et al. Research on Comprehensive Optimization Based on Grey Association for Evaluating the Operational Effectiveness of Weapon System. Fire Control and Command Control, Vol. 32, No. 11, pp 85 -87(2007)

3. Chang Shuang-Jun, Ma Jin-ya. Application Study on Synthetical Optimization Based on Grey Associated Method for Evaluating Effectiveness of Weapon system. Journal of Sichuan Ordnance, Vol. 32 May. Pp.16 -19 (2011)

4. Wang Haibin, An Improved Analytic Network Process Effectiveness Evaluation Method Based on Grey Relational Analysis. Missiles and Space Vehicles, No. 5, pp. 66-69(2013).

5. Chen Qin, Zhao Yu-hui, Du Jun. “Application of the Improved Grey Correlation Analysis on Evaluating Radar Low Proability of Interception Performance “. Fire Control Radar Technology, Vol. 41, No.2, Jun. pp. 13 -16(2012)

6. ChihHsuan Wang. A systematic approach to select the optimal project portfolios for green manufacturing: An empirical study on TFT-LCD fabrication processes. Proceedings of the 2015 International Conference on Industrial Engineering and Operations Management Dubai, UAE, March 3 - 5, (2015)

7. Zhang Shi-fang. Several Method for Fuzzy Multiple Attribute Decision-making with Applications, Xidian University, (2012)

8. Xu Rui-li, Xu Ze-shui. Study on the practice and recognition of Mathematics in Practice and Theory, Vol. 37, No. 24, Dec, pp. 1 -7(2007)

9. Chen Chun-fang, Zhu Chuan-xi. Method of Interval Number Ranking Based on Vector Similarity and Application. Statistics and Decision, No. 3, pp 76-78(2014)

10. Zhang Shi-fang, Liu San-yang.A GRA-based intuitionistic fuzzy multi-criteria group decision making method for personnel selection, Expert Systems with Applications, 38(9) pp. 11401-11405 (2011)

11. Wu Jiang, Huang Deng-shi. Review of the Research on the Method of Interval Number Ranking. Systems Engineering, Vol. 22, pp.1-4(2004)

12. Xu Shihong,Zhao Weibin. Study on the Effectiveness Evaluation Method Based on the Combination of AHP and Grey Interval Correlation. Journal of Electrical and Electronic Engineering. Vol.3, No.5, pp177-180 (2015).

13. Niu Dezhi, Chen Changxing, et al. Effectiveness Evaluation for Aircraft and Combat Application based on Data Link. Application of Electronic Technique. Vol. 40, No. 7, pp. 103-105(2014)

14. Zhou Lei, Zhang Hanyi, et al. Static check of WS-CDL document. IEEE International Symposium on Service-Oriented System Engineering, No. 8, pp 142-147(2008)

15. Zeng Guo-qi, LI Siyin,et al. Simulation Research on Satellite Data Link Communication of the Rocket Borne General Character Phased Array. Application of Electronic Technique. Vol. 38, No. 11, pp 104-107(2012)

16. CHEN Qiang et al. Effectiveness Analysis Evaluation on Force in Air Combat Process Based on Data Link. JOURNAL OF AIR FORCE ENGINEERING UNIVERSITY. 2015, No. 3, pp. 16-20

17. MA Xia. Performance analysis and simulation of tactics datalink relative navigation. Journal of Computer Applications. Vol. 31, No. 1, pp.165-167(2011)

18. QIAO Yong-jie, SUN Liang, CHEN Xing-bo. Effectiveness Evaluation of Antimissile-warning-system Based on Operational Activity [J]. Fire Control \& Command Control. Vol. 36, No. 6, pp 104-107(2011)

19. MA qian. Efficiency Evaluation Method of Data Link Network Based on Grey Relational Analysis. Technology Innovation and Application. Vol. 3, No. 24, pp. 30-32(2015) 